\title{
Towards dynamic simulation of a whole cell model
}

\author{
Jae-Seung Yeom Konstantia Georgouli Robert Blake Ali Navid \\ \{yeom2,georgouli1,blake14,navid1\}@llnl.gov \\ Lawrence Livermore National Laboratory \\ Livermore, California, USA
}

\begin{abstract}
Whole-cell models (WCMs) aim to integrate the sum of our knowledge about the mechanistic processes of an organism that are inherently multi-scale and dynamic. Such comprehensive models would enable us to address many challenging questions, such as understanding interactions and coupling between pathways, examining system properties, and identifying gaps in our biological knowledge. WCMs integrate a diverse array of intracellular pathways through an equally diverse assortment of computational methods. Among these methods, stochastic simulation helps implement the most detailed model. However, it is also the most time consuming to execute. Furthermore, WCMs involve some of the largest known biochemical reaction networks. To speed up the simulation and accelerate the development of such a model, we present a parallel implementation of stochastic simulation algorithm (SSA) and its application to a whole cell reaction network.
\end{abstract}

\section{CCS CONCEPTS}

- Computing methodologies $\rightarrow$ Simulation support systems.

\section{KEYWORDS}

Whole-cell Modeling, Stochastic simulation algorithm, SBML, Highperformance computing

\section{ACM Reference Format:}

Jae-Seung Yeom Konstantia Georgouli Robert Blake Ali Navid. 2021.

Towards dynamic simulation of a whole cell model. In 12th ACM International Conference on Bioinformatics, Computational Biology and Health Informatics (BCB '21), August 1-4,2021, Gainesville, FL, USA. ACM, New York, NY, USA, 10 pages. https://doi.org/10.1145/3459930.3471161

\section{INTRODUCTION}

The complexity and scope of in silico models of biological processes have continually increased. Advances in high-throughput technologies permit systems-level analyses of complex biosystems (including humans) that result in generation of massive amount of omics data. Extracting new insights from this data necessitates development of computational models that can simulate these complex biological processes in its entirety requiring us to combine

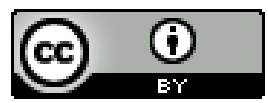

This work is licensed under a Creative Commons Attribution International 4.0 License. BCB '21, August1-4, 2021, Gainesville, FL, USA

(C) 2021 Copyright held by the owner/author(s).

ACM ISBN 978-1-4503-8450-6/21/08.

https://doi.org/10.1145/3459930.3471161 different modeling techniques. For example, informing macroscale models with cellular metabolic information requires combining results from genome-scale methods such as flux balance analysis (FBA) with ordinary differential equation (ODE)-based models that can predict the transient changes in a system. Similarly, simultaneously accounting for rare and frequent biological events requires combining ODE and SSA-based models. Common model building practices such as combining complex multistep processes into a single reaction present challenges that require expert input in order to parameterize the models so that they can be simulated using available solvers.

Developing genome-based whole-cell models (WCMs) that integrate the above modeling methodologies for analysis of unicellular organisms is a first step toward describing and simulating complex multi-cellular organisms or ecosystems. Development of WCMs for even small systems is a time consuming and labor intensive process that will greatly benefit from tools that can speed up such a process. Among the common biological modeling methods, SSA deals with individual molecular counts and is therefore the most detailed. This also means that it is the most time consuming to execute. In this paper we present our effort aimed at tackling two issues associated with current state of the art biological modeling of complex systems: 1) simulating SSA in a manner that permits its use for simulating biology scale set of reactions and b) devising parameterization schemes that would allow for us to test our platform using WCMs.

In particular, we make the following contributions:

- A built-in just-in-time (JIT) method to ingest systems biology markup language (SBML)-based models as inputs with arbitrary rate expressions without relying on any specific JIT framework.

- A method to analyze implicit dependencies among species, reactions, and transient parameters, and to restructure the network for exposing dependencies while parallelizing the network.

- A parallel implementation of a stochastic simulation algorithm.

- The composition of representative workloads of a large biochemical reaction network.

- The first parallel stochastic simulation of a whole cell's reaction network.

Following the introduction, Section 2 provides the background information. Section 3 discusses the design decisions made to ingest an input model and to process it in parallel. Section 4 describes how we implement the solutions. Section 5 presents a set of performance evaluation results. Section 6 reviews the existing frameworks that implement stochastic simulation and supports the SBML-based input. 


\section{BACKGROUND}

In this section, we provide a high-level overview of whole-cell modeling, the modeling technique SSA which is one of the methods used in WCM simulations, and the Systems Biology Markup Language (SBML) which can be used to define WCMs.

\subsection{Whole-cell modeling}

Revolutionary advances in high throughput analytical methods have led to paradigm shift in biological sciences. Instead of the "reductionist" focus of studying specific biological processes, holistic catalogs of biological components of a biosystem are generated and this information allows for analyses of organisms as a complete system. System-level analyses of biological systems have resulted in drastic breakthroughs in the fields of medicine and synthetic biology. Given the massive amounts of data that need to be analyzed for systems biology studies, use of state-of-the-art computational models is an absolute necessity. Although various types of in silico genome-scale modeling of biological systems have been attempted, until recently none of these methods accounted for the activity of all the known components of a biosystem. In each case processes of interest were simulated in great detail using the most appropriate mathematical methods while the rest of the system would be generalized in a manner that would lack the details needed to use the models for prediction of system-wide outcomes following genetic or environmental perturbation. Furthermore, use of different modeling languages and mathematical formalisms for different models prevented assembly of unified models that could utilize the knowledge collected for development of earlier models. That is why to date only one truly WCM of a biosystem has been developed [13]. The novel hybrid approach used to develop this WCM divides disparate cellular processes into numerous modules where best modeling formalisms are used for simulating activity of each bioprocess. Using a method akin to discrete events simulation, the modules are run based on their state at the time of each event. Results from each module that affect the state of other modules are transferred to appropriate source and appropriate updates are made prior to next simulated event. Although this novel hybrid modeling method allows for generation of WCMs, the simulated systems have thus far been very simple. Systems of significant medicinal or industrial importance generally tend to be very complex and use of the hybrid method as initially devised would not work for simulating the workings of these systems. To simulate WCM for complex multi-cellular systems requires taking advantage of highperformance computing (HPC) that would allow simulations of multi-scale and highly compartmented models in a parallel fashion.

\subsection{Stochastic simulation algorithm}

Biochemical reactions are the building blocks of WCM. Each reaction represents a discrete event that converts a minimal set of constituents of particular chemical species and produces new one(s). Various methods exist to simulate such a model, offering the exact solution to represent the individual changes in countable constituents. Stochastic simulation algorithm (SSA) is a method to simulate statistically correct trajectories of the time evolution of such a model in terms of the species population in a well-mixed volume [20]. Use of SSA is necessary for accurate quantitative simulation of reaction dynamics involving species with low constituent counts, while being the most computationally intensive mode of simulation.

In addition to the direct method (DM) originally proposed by Gillespie [20], there exist multiple variants of the SSA method [1, 8, $9,19,30]$. Among these, the next reaction method (NRM) employs a dependency graph to identify the coupling between reactions via their commonly referenced species, and to selectively update the propensity and the time of the next occurrence of each reaction impacted by firing one [19]. It also relies on the indexed min-heap to selectively refresh the priority queue. These modifications improve the performance. The most important characteristic is, however, that it exposes further parallelization opportunity as it independently computes the time of next occurrence of each reaction. On the other hand, the direct method first computes the time of the next firing in the whole system based on the current propensity spectrum, and then determines which reaction that will be. The optimized direct method (ODM) enhances DM by adopting the update dependency graph (UDG) in NRM [9]. The sorting direct method (SDM) maintains the list of propensities ordered by the magnitude to reduce search cost [30]. Section 3.3 further discusses our approach to parallelize NRM.

\subsection{Systems Biology Markup Language}

Our framework takes advantage of the Systems Biology Markup Language (SBML) [16] to describe a model for WCM simulation. SBML is a widely supported xml-based language for representing and exchanging models of biological systems of different complexity. This high-level model format offers all the necessary elements and features for the representation of a wide range of dynamical models which can be simulated by using ODEs and stochastic simulations. Specifically, the relatively recent development of several new SBML extensions known as "packages" has enabled the support of constraint-based, logical, spatial, rule-based and composite models [24]. Majority of these types of modeling are used in the hybrid method for generating WCMs. In addition, SBML model specifications are implementation independent enabling extensibility, portability and reusability. This would allow WCM developers to use the large databases previously developed SBML-based models (e.g. the BioModels database [17]) as building blocks for their new models. All these aforementioned facts make SBML the most suitable format for describing whole cell models.

\section{APPROACH}

\subsection{Graph-based representation}

We represent a biochemical system focusing on reaction kinetics with a bipartite graph that consists of the vertices of two different types, the species and the reaction, and the directed edges connecting the two different types of vertices [36]. An edge coming out of a reaction type vertex that connects to a species vertex indicates that the species is a product. An edge going into a reaction vertex from a species vertex means the species is a reactant. We assign a stoichiometry number to each edge. Each vertex has a set of attributes. A species vertex is characterized by a copy number while a reaction vertex has a user-defined function that computes its reaction 
rate. For handling heterogenous types of vertices with an elaborate set of attributes and the operations on them, we employ an object-oriented graph-based data structure that improves extensibility. However, a representation based on a matrix of an elementary value type or a plain-old-data structure would have allowed further performance optimizations at the cost of generality [23].

\subsection{General reaction rate expressions}

To derive the dynamics of the chemical reaction network, mass action kinetics are generally used [40]. For example, consider the following bimolecular reaction where compounds $A$ and $B$ are consumed to produce compound $C$

$$
\alpha A+\beta B \rightarrow \gamma C
$$

where $\mathrm{A}, \mathrm{B}$, and $\mathrm{C}$ are three species with concentrations a, b, c, and stoichiometric coefficients $\alpha, \beta, \gamma$. The stoichiometric coefficients are positive numbers expressing how much of the species involved in the reaction. Based on mass action kinetics, the reaction rate (rr) equation is

$$
r r=k a^{\alpha} b^{\beta}
$$

where $k$ is the reaction constant or coefficient.

Mass action kinetics methods are mainly representing deterministic biochemical systems where a large number of molecules are well mixed and distributed homogeneously [38]. However, some bioprocesses cannot be solved deterministically and need to be treated as stochastic biochemical systems. These processes are generally those where the number of molecules of reactants are relatively small; or when the bioprocess of interest occurs on rare occasions (such as a genetic mutation that results in oncogenesis). This is why in case of cancer biochemical models, a significant fraction of the reactions have non-mass action reaction rate formulas [11]. For these reasons, our framework provides the flexibility to define reaction rate formulas by using arbitrary functions where mathematical functions, state variables, and parameters can be used. For instance, the arbitrary expression for the above example is

$$
r r=f(k, a, \alpha, b, \beta)
$$

Our framework relies on the SBML Level 3 for the representation of input model. libSBML is an application programming interface performing the lexical and syntactic analyses of a SBML model. Biological network information such as constituent species and reactions are extracted by using the libSBML [6] and converted into an internal representation.

In the early development stage, the evaluation of the reaction rate formulas was carried out by means of the $\mathrm{C}++$ Mathematical Expression Toolkit Library (ExprTk) [35]. ExprTk library can parse and evaluate mathematical expressions as strings in a specific format. Specifically, the input to this tool should follow specific expression structure for using advanced features and capabilities. For example, the following structural textual string can be used to define a kinetic reaction rate expression.

$$
\text { string } r r \_s t r=\text { "var } k:=0.005 ; r r:=k * Y *(Y-1) / 2 ; "
$$

This is a multi-statement ExprTk expression composed of a variable definition and initialization and a mathematical expression of the reaction rate formula. $Y$ is the count of species $Y$ which has been stored to the symbol table of ExprTk. We generate such textual representations of expressions obtained from SBML in the ExprTk syntax. ExprTk stores the abstract syntax trees of the expressions and uses recursive descent to evaluate them. This process involves multiple function invocations to compute a reaction rate.

Currently, to speed up the simulations, our simulator generates $\mathrm{C}++$ code that is dynamically compiled and linked to the application after parsing the SBML file. Section 4.2 discusses the details of code generation. Section 5.1.3 presents a performance comparison of the two methods based on ExprTk and code generation.

\subsection{Identification of parallelism}

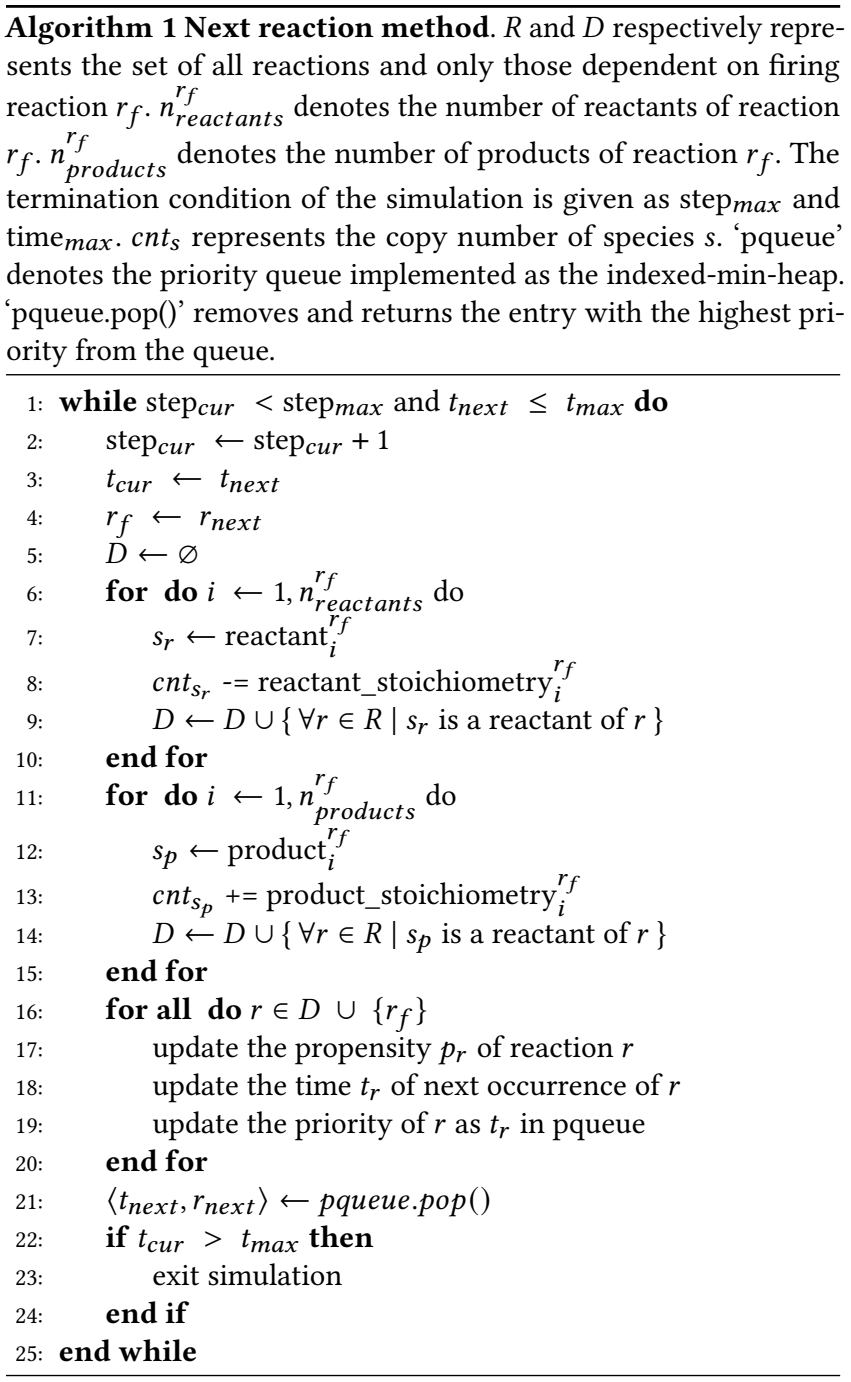

Here, we identify the parallelism in NRM that exists at different levels of work granularity depending on the characteristics of the problem. At the top level, we expose the parallelism by splitting the given reaction network into multiple partitions. This is especially effective on large networks. In Section 6, we discuss even more coarse-grained approaches in existing works, which is to simultaneously execute multiple realizations of a system. 
At a finer level, there exist multiple parallelization opportunities. If a network exhibits a high degree of reaction node, i.e., has a large number of reactant or product species associated with a single reaction, we may simultaneously update population of reactants and/or products in executing a reaction (line 6-15 in algorithm 1). This approach is, in fact, generally applicable to various SSA algorithms including DM and NRM. This portion of the code mostly generates the cost to access the underlying graph data structure.

Another one specific to NRM is to simultaneously update dependent reactions, especially if a network shows a high degree of species node (lines 16-20 in algorithm 1). The line 17 generates the major arithmetic computational load that computes the propensity using the reaction rate formula in addition to the cost to access the states of reactant and modifier species. Section 3.2 describes how we allow users to express an arbitrary form of a reaction rate formula. Section 4.2 discusses how callable functions are created from user inputs, and Section 5.1.3 evaluates the performance of calling the functions. The line 18 updates the time of the next occurrence of each reaction being updated based on the latest propensity computed at line 17. This operation differs for $r_{f}$ and for the rest of reactions. In case of the former, a random number $g$ is used to compute $t_{r_{f}} \leftarrow t_{c u r}+1 / p_{r_{f}} \ln (1 / g)$. For the latter, the update performs $t_{r} \leftarrow\left(p_{r}^{\text {old }} / p_{r}^{\text {new }}\right)\left(t_{r}-t_{\text {cur }}\right)+t_{\text {cur }}$ if $p_{r}^{\text {old }}>0$. Otherwise, it is updated similarly to the former.

Line 19 updates the priority of the reaction $r$ by first locating where it is in the queue, replacing the priority (i.e., time), and reorder the queue to maintain the property of the priority queue. Locating an item in a min-heap requires a separate indexing structure which is automatically updated as the location of an item changes in the queue. The update takes the time cost of $O\left(n_{r}\right)$, where $n_{r}$ is the number of reactions.

\section{IMPLEMENTATION}

\subsection{Simulation infrastructure}

We implement our simulation infrastructure in $\mathrm{C}++$, which is available at [41]. At startup, it 1) loads an input SBML file using libSBML, 2) constructs a reaction network using the information obtained by querying the model via the libSBML interface, 3) generates the machine code for reaction rate formulas, and finally 4) initializes the species population and the initial reaction propensities.

The network data structure is based on the BidirectionalGraph concept in boost::graph library, which supports the directional graph with the efficient access to in-edges. Each graph node has a set of attributes such as the label, the type and the partition index, and is associated with a polymorphic property object. For a reaction type node, the object contains a callable function of its rate formula. For a species type node, it contains the copy number of the species.

As with LOCAT [23], our NRM implementation relies on a bipartite update dependency graph (BUDG). This differs from the original NRM where a dependency graph has only the reaction type vertex. BUDG bounds the maximum degree to an order of connected species rather than coupled reactions in a dependency graph. This is especially beneficial for large networks where some species are involved in many reactions. For example, in an extreme case where there exists a species that is connected to all of $n_{r}$ reactions of the system while a reaction is connected to at most $d_{r}^{\max }$ species, the total connectivity of such a dependency graph becomes $O\left(n_{r}^{2}\right)$ without exploiting the bipartite structure, and $O\left(n_{r}\right)$ with BUDG as $d_{r}^{\max }$ is a constant. Furthermore, our reaction network itself serves as the dependency graph with no additional data structure. We implement not only NRM, but also DM as well as the sorting optimized direct method (SODM) which takes advantage of BUDG $[9,19,20,30]$. However, currently only NRM is parallelized.

The infrastructure offers two trajectory recording mechanisms, tracing and sampling. The former records every stochastic event during simulation. The latter records the accumulate population changes at a user-defined interval in terms of the simulation time or the number of events. Recorded events are first stored into a memory buffer to minimize file I/O during simulation for better performance. When the buffer is filled up, the buffer content is serialized and flushed into a temporary file. At the end of simulation, all the temporary files are read one by one to reconstruct the whole trajectory while writing it into a human readable file.

As a general user interface other than the SBML-based modeling interface, we adopt Google's protocol buffer language [21]. This framework allows us to define a succinct and human readable nested structure to describe simulation setup details without the burden of implementing the parser.

To avoid on-line compilation cost with a large model, we offer the capability to reuse a pre-built library that is generated in a previous execution or by an offline tool we offer. The source code generated for the model used in this study contains more than 2.1 million lines. Our JIT method relies on parallel compilation to speed up, of which detail is discussed in Section 4.2.

\subsection{Code generation}

Code generation is used to compute kinetic reaction rate formulas mentioned in Section 3.2. Each formula is compiled into a separate function with a dynamic symbol. Each function can then be invoked from within WCS as if it was natively compiled into the application. This JIT compilation requires only a working $\mathrm{C}++$ compiler and dynamic linking. The fact that there is no need for further dependencies enables the code portability and reusability of our framework. Specifically, the steps for the code generation are:

(1) After the SBML model has been ingested and analyzed, $\mathrm{C}++$ code is generated into temporary files. Variable dependencies are found by performing a pre-order traversal of the Abstract Syntax Tree (AST) representation of the mathematical expression of the reaction rate formula. A function is created for each reaction to calculate its rate formula, and each function is marked as part of the externally visible interface. A dynamically linked library is compiled from the temporary $\mathrm{C}++$ files using the compiler on the target platform.

(2) The dynamically loaded library is opened using the dlopen function of the programming interface to the dynamic linking loader. The address in which a symbol for a reaction is loaded into memory is returned by calling the dlsym function. Thereafter this address is utilized for the evaluation of the kinetic reaction rate of this reaction.

Code generation offers a great flexibility in supporting all the SBML components. 


\subsection{Transient parameters}

Special care was taken to represent transient parameters within our graph-based data structures. A transient parameter is any parameter who's value depends on other species. In some cases, a transient parameter serves as a temporary variable that allows breaking down a complex calculation into multiple steps, the result of each is stored into this intermediate variable. In other cases, it represents a simulation state that depends on other quantities, such as the counts of certain species, other state variables, and/or global constants. In case of the former, it is local. In case of the latter, it can be shared in different reaction rate computations. For example, consider a reaction in which the counts of two reactants species $(S 1$ and $S 2$ ), one modifier species $M 3$ - a species is neither consumed nor produced -, the value of one simulation state variable $P 1$, and a global constant $G 1$ are appeared in the kinetic reaction rate formula of this reaction. $P 1$ is a transient parameter that its value depends on the counts of two species ( $S 4$ and $S 5$ ) and the value of another transient parameter $P 2$ which in turn depends on $S 6$.

$$
\begin{aligned}
S 1+2 * S 2 \rightarrow S 7 & : f(S 1, S 2, M 3, P 1, G 1) \\
P 1 & =g(S 4, S 5, P 2) \\
P 2 & =h(S 6)
\end{aligned}
$$

A naïve representation of these transient parameters in partition graph would require the addition of another type of vertex apart from the existing two types, species and reactions. Figure 1 shows the graph-based representation after the addition of the parameters. Moreover, the hypothetical related generated code is presented in this figure. The green color vertices indicate the required new type of vertex, the transient parameters. The value of a parameter vertex would be evaluated based on the inputs of species, other parameters and the current value of itself. Nevertheless, this approach would complicate the partitioning and have a possible impact on parallelization during the evaluation process.

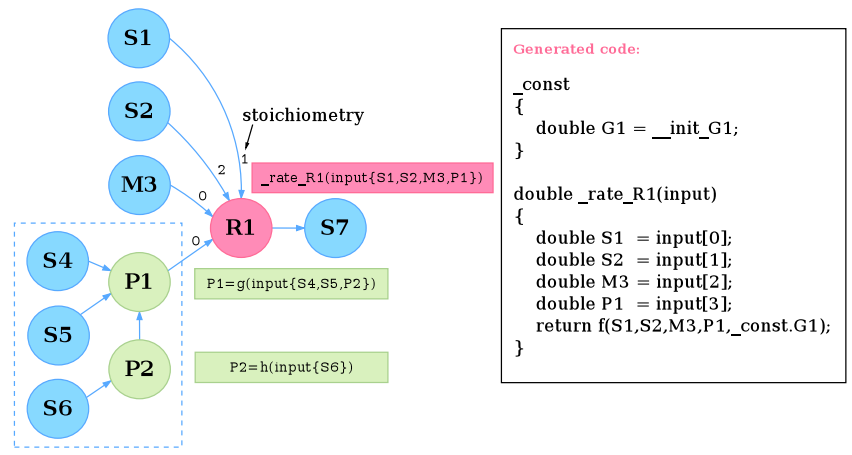

Figure 1: Naïve representation of transient parameters.

To overcome these limitations, the network and reaction rate functions were flattened to eliminate these transient parameters. The graphical representation of this approach and the corresponding generated code are shown in Figure 2. After flattening there is no need for a new parameter type of vertex and therefore the partitioning complexity remains mainly unchanged. This approach is as general as representing each transient parameter separately in the graph data structure. As shown in Figure 2, the input species of the evaluation functions for the transient parameters $P 1$ and $P 2$ are appended to the inputs of the reaction $R 1$ since their evaluation of the transient parameters occur inside the generated code. These appended input species are treated as modifiers of the reaction $R 1$ to express the dependency relationships, but to prevent $R 1$ from modifying their states. In other words, they are connected to $R 1$ as if they are reactants but with stoichiometry value 0 . Hence, this approach gives an efficient solution for the handling of the transient parameters and has great potential for more complicated models.

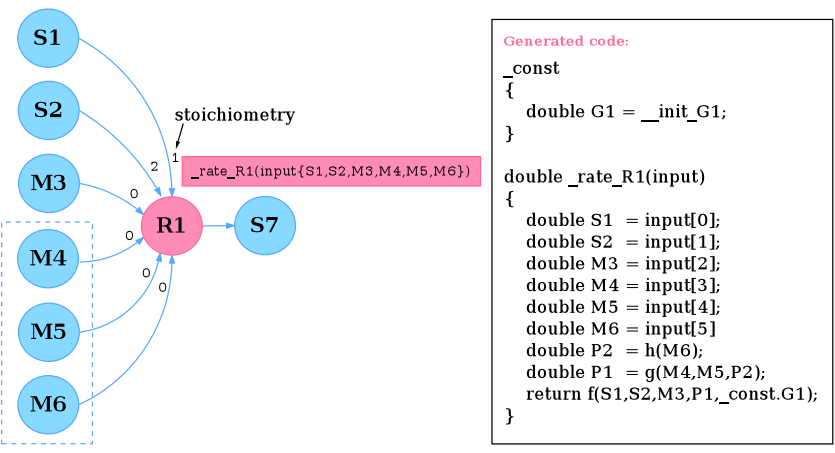

Figure 2: Exposing the hidden dependencies of transient parameters to facilitate parallelization.

\subsection{Parallelization}

We take advantage of the OpenMP parallelization constructs on the multiprocessors with shared memory architectures [5]. Algorithm 2 parallelizes the sequential NRM algorithm shown in Algorithm 1. It can exploit up to two levels of nested parallelism. At the top level, the while loop at line 1 runs in parallel over different graph partitions. Specifically, the body of the loop is enclosed in an OpenMP parallel block. Depending on the problem, the second level can be selectively enabled for loops between the lines 6-10, the lines 11-15, and the lines 16-20. The parallelization at the first level resembles the parallelization on a distributed memory system except that the reduction at line 22 is performed on a shared memory system. In the rest of the section, we refer 'local' data as that are exclusive at the first level, but shared in the second level among threads.

As the first step, we rely on a graph partitioner to generate a list of partition index, by which we identify which partition each vertex belongs to. Each OpenMP thread creates its own copy of the entire network with a private set of RNGs and marks each vertex with the partition index of the vertex. We plan to apply memory footprint compression by deallocating the property objects of non-local vertices that are not connected to any of local vertices. Each thread performs the same operations as with the sequential execution in Algorithm 1 except on lines 9, 14 and 21. For identifying dependent reactions at lines 9 and 14, it checks if a candidate reaction belongs to the partition it manages. If so, it is added to the set $D^{\text {local }}$. Otherwise, it is ignored.

The loop between the lines 16-20 involves operations on a local priority queue. As the non-local reactions are filtered, the size of the queue as well as the amount of work performed on it is bounded 
Algorithm 2 Parallelized next reaction method. $R^{\text {local }}$ and $D^{\text {local }}$ respectively represents the set of all local reactions and only those locally dependent on firing reaction $r_{f} . n_{\text {reactants }}^{r_{f}}$ denotes the number of reactants of reaction $r_{f} . n_{\text {products }}^{r_{f}}$ denotes the number of products of reaction $r_{f}$. The termination condition of the simulation is given as step $\max$ and $t_{\max }$. cnt $t_{s}$ represents the copy number of species $s$, which is globally coherent. 'pqueue local' denotes the local priority queue implemented as the indexed-minheap. 'pqueue local.pop()' removes and returns the entry with the highest priority from the local queue.

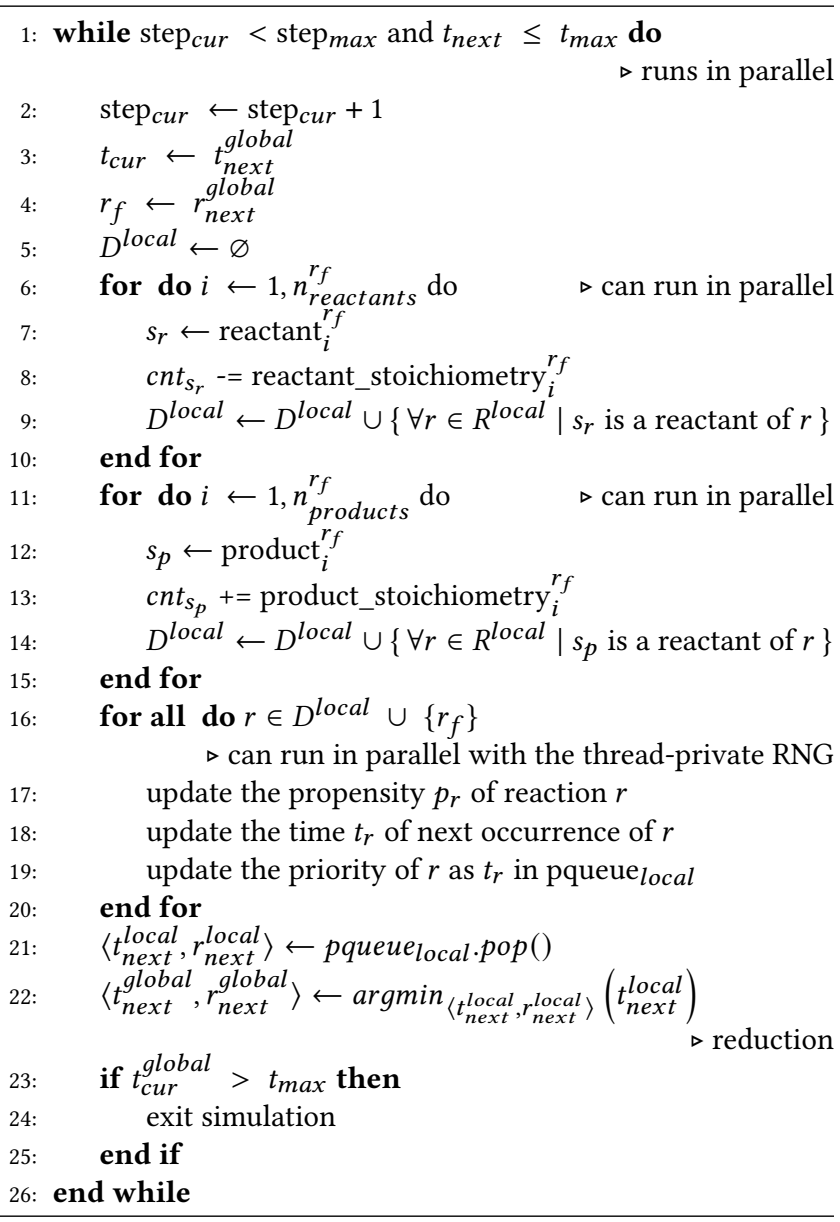

by $O\left(\log \left(n_{r}^{\text {local }}\right)\right)$, where $n_{r}^{\text {local }}$ is the number of local reactions. This queue is a shared data structure within the second-level loop. Therefore, it is declared as a critical region for parallelizing the loop using OpenMP. The parallelization at the first level helps reducing the amount of work to be done in this critical region.

The loops between the lines 6-10 and the line 11-15 can run with a dynamic loop schedule in parallel execution. On the other hand, the loop between the line 16-20 may rely on a random number generator (RNG) at the line 18, and thus, the scheduling policy needs to be carefully chosen to affirm the reproducibility and the correctness. The pairing between the loop index and the RNG instance must be identical across different executions given the same seeding of RNG instances. Moreover, processing of different loop indices should not overlap in time but execute in a consistent order if they map to the same RNG instance. We achieve this by relying on a static loop scheduling and thread-private RNGs.

Note that the data structure used to represent $D^{\text {local }}$ is std: : set which is not a randomly accessible container. Therefore, to allow an OpenMP thread to directly map to the data corresponding to the loop index it is about to process, the content of the set is copied to a random-access container (e.g., vector) object before entering the loop. This incurs an overhead cost that does not exist in the sequential code. Alternatively, we may cache the result of such a conversion for each reaction. This, however, revokes the advantage of BUDG discussed in Section 4.1. Line 21 performs a local operation to obtain a candidate for the next event, followed by a reduction operation (line 22) to identify the globally earliest reaction.

\subsection{Synthesizing representative workloads}

While the ideal workload to demonstrate the capabilities of our framework would be the only existing whole cell model [13], the current format of its representation requires non-trivial changes to use it directly for our purpose. Instead, we take advantage of a recently published adaptation of the same WCM [7]. This model has been constructed to enable qualitative analyses such as assessing the topology of the network and its robustness to genetic perturbations. However, it is not suited for simulating the system's dynamics because it lacks critical chemical kinetics parameters. To generate a realistic workload for stochastic simulations of WCM, we used the generated network in [7], and estimated the parameters for the kinetic reaction rates by relying on our biological modeling experience. With this, our intention was not to complete a predictive model, but to instead enable performance investigation with 'computationally plausible' workloads. In this section, we discuss how we generate a rate formula for each reaction in the Mycoplasma genitalium (MG) network [7].

The model [7] consists of fifteen cellular processes that combine to form a single component model. The reason why we cannot use common mass action kinetics for all the reactions in the model is that like many other 'system-level' models, the MG model has truncated large and complicated reaction pathways into single reactions, producing reactions with unusually large stoichiometry coefficients (see Table 2). These complex reactions usually describe behavior of large (de)polymerization processes. For example, the set of reactions: $P C+N_{1} \rightarrow P C N_{1} ; P C N_{1}+N_{2} \rightarrow P C N_{1} N_{2} ; P C N_{1} N_{2}+N_{1}$ $\rightarrow P C N_{1} N_{2} N_{1} ; P C N_{1} N_{2} N_{1}+N_{1} \rightarrow P C N_{1} N_{2} N_{1} N_{1} ; P C N_{1} N_{2} N_{1} N_{1}+N_{3} \rightarrow$ $P C N_{1} N_{2} N_{1} N_{1} N_{3} ; P C N_{1} N_{2} N_{1} N_{1} N_{3}+N_{1} \rightarrow P C N_{1} N_{2} N_{1} N_{1} N_{3} N_{1}$ will be modeled as $P C+4 N_{1}+N_{2}+N_{3} \rightarrow P C N_{1} N_{2} N_{1} N_{1} N_{3} N_{1}$. While in this example calculating the reaction rate is computationally tractable (rate $\left.=k\left[N_{1}\right]^{4}\left[N_{2}\right]\left[N_{3}\right]\right)$, for generation of long polymers like proteins, DNA, and RNA, the large stoichiometric coefficients for polymer subunits in these reactions (typically $>100$ ) prevented us from using simple mass action kinetic rates.

To overcome this problem, we have devised three approaches for constructing reaction rate formulas to represent all the processes in the model. With the simplifying assumption of a $\mathrm{pH}$-neutral reaction environment, we removed $\mathrm{H}_{2} \mathrm{O}$ and $\mathrm{H}^{+}$from the list of species. Table 1 shows the distribution of the reactions based on the three 
Table 1: Distribution of Mycoplasma genitalium reactions across the three different approaches.

\begin{tabular}{crr}
\hline Approach & $\begin{array}{r}\text { Num of } \\
\text { species }\end{array}$ & $\begin{array}{r}\text { Num of } \\
\text { reactions }\end{array}$ \\
\hline i & 36,521 & $66,678(81 \%)$ \\
ii & 5,700 & $5,652(7 \%)$ \\
iii & 15,478 & $10,328(12 \%)$ \\
\hline Total & 37,028 & 82,662 \\
\hline
\end{tabular}

approaches. Table 2 indicates some examples of reactions covered by each approach. It is important to note that all the reactions in the model are irreversible, i.e., reversible reactions have been modeled as two irreversible reactions.

For the discussion that follows, let $R_{j}$ be the set of its reactants of reaction $j$. The reaction may also involve a set of modifiers, $M_{j}$. $[s]$ denotes the millimolar concentration of species $s$, and $\min _{c}[S]$ is the minimum concentration of a species in set $S$.

4.5.1 Approach $i$. This approach covers the broadest set of reactions in the model ( $81 \%$ of model reactions). These are all the reactions with $\sum_{s \in S_{j}} \sigma_{s}<5$, where $\sigma_{s}$ is the stoichiometric coefficient for reactant $s$ or 1 if $s$ is a modifier. We chose 5 as the threshold to use the mass action kinetic for production of short nucleotide sequences (length of chain $<5$ ) and accounting for concentration of the 4 nucleotide types. Let $S_{j}=R_{j} \cup M_{j}$ and $k_{j}$ be the reaction coefficient of reaction $j$. We define the rate for reaction $j\left(v_{j}\right)$ as:

$$
v_{j}=k_{j} \cdot \prod_{s \in S_{j}}[s]^{\sigma_{s}}
$$

4.5.2 Approach ii. This approach is for reactions of RNA and protein degradations, where long polymer chains are deconstructed into their constituent components-nucleotides (nuc) for mRNA and DNA and amino acids (AA) for proteins. To ensure that the longer chains break down slower than shorter chains, we linked the rate coefficient of degradation to the length of polymer (LP). LP was calculated by summing the stoichiometric coefficients of the polymer subunits as product species. Although the rate of protein and mRNA degradation is dependent on the composition of the polymer [10] and varies significantly between different organisms [32], in absence of quantitative values for $M$. genitalium, we used a value of $5(\mathrm{nuc} / \mathrm{sec})$ and $0.5(\mathrm{AA} / \mathrm{sec})$ for speed of polymer degradations for mRNAs and proteins respectively. These values are loosely based on the average degradation times of 5 and 40 minutes, and average polymer lengths of 924 and 300 for mRNA and proteins respectively in prokaryotic organisms $[15,31]$. Let $E$ be the set of nucleotide triphosphates like ATP and GTP that provide the energy needed for protein degradation, $E_{j}=\left\{s \mid s \in R_{j} \cap E\right\}$ and $S_{j}=R_{j}-E_{j}$. Also, let $\mathbb{X}_{j}=\left\{M_{j}, E_{j}\right\}-\{\emptyset\}$ and $k_{j}^{m i n}$ be $\prod_{X \in \mathbb{X}_{j}} \min _{c}[X]$. We finally define the rate for reaction $j$ as:

$$
v_{j}^{d e g}=k^{d e g} \cdot k_{j}^{\min } \cdot\left(\prod_{s \in S_{j}}[s]^{\sigma_{s}}\right)
$$

where $k^{d e g}$ is $5 / L P_{m R N A}$ for RNA degradation and $0.5 / L P_{\text {prot }}$ for protein degradation.

4.5.3 Approach iii. Polymer chain elongation processes such as translation, transcription and DNA replication consist of reactions where a large number of reactants are combined to form significantly fewer number of products. To account for the complexity, and large number of stoichiometric coefficients in these kinds of reactions, we devised a third approach for calculating the reaction rate. To this end, let $N$ be the set of nucleotides and deoxynucleotides, $A$ the set of all 20 common amino acids and $U$ the set of all other polymerization subunits. For reaction $j$, we define $N_{j}=\left\{s \mid s \in R_{j} \cap N\right\}, A_{j}=\left\{s \mid s \in R_{j} \cap A\right\}$ and $U_{j}=\left\{s \mid s \in R_{j} \cap U\right\}$. We then define $S_{j}=R_{j}-\left(N_{j} \cup A_{j} \cup U_{j}\right), \mathbb{X}_{j}=\left\{M_{j}, N_{j}, A_{j}, U_{j}\right\}-\{\emptyset\}$ and $k_{j}^{\text {min }}=\prod_{X \in \mathbb{X}_{j}} \min _{c}[X]$. As with the depolymerization processes, we ensured that the rates reflect the length of the polymer being generated by scaling the rate coefficients. We define the rate of reaction $j$ as:

$$
v_{j}^{p o l}=k^{p o l} \cdot k_{j}^{\text {min }} \cdot\left(\prod_{s \in S_{j}}[s]^{\sigma_{s}}\right)
$$

wheren $k^{p o l}$ is $20 / L P_{\text {prot }}$ for translation, $60 / L P_{m R N A}$ for transcription [33], and $1000 / L P_{D N A}$ for DNA replication [2].

Aside from polymerization reactions, there are a few other reactions in the model, such as sequential phosphorylation processes that have also been truncated as single reactions. For these processes we also use the same formula as that for the polymerization process. The $k^{p h o}$ values for these processes were $0.05 /$ number of phosphorylation events [4].

\section{RESULTS}

5.0.1 Experimental setup. We present results from experiments on a machine that has total 192 GB memory and two sockets of the Intel Xeon CLX-8276L processor with total 56 cores, operated by linux with kernel 3.10.0. The hardware threading was disabled. We used gcc 8.1 .0 with the option '-march=native', '-mfpmath=sse' and '-Ofast' to compile both our code and COPASI (version 4.30). The performance was measured only for the simulation loop excluding model loading. We used the boost library version 1.69.0, the SBML library version 5.16.0, and the Metis library version 5.1.0.

\subsection{Performance evaluation}

5.1.1 Parallel execution. Figure 3 shows the performance comparison between the parallel implementation and the sequential one using the entire MG network. The former replies on OpenMP with which each thread runs the while loop in Algorithm 2 on each network partition obtained by Metis [25]. We set the OpenMP environment variable OMP_PROC_BIND to spread, and let each thread allocate and initialize its own network to take advantage of the first-touch data placement policy. The objective function of Metis was set to minimize the maximum degree between sub-domains to allow further flexibility in satisfying the load balancing constraint. We ran ten runs of 70,000 simulation steps with varying number of threads. Figure 3 shows the average of each case. We observe $10.3 \mathrm{x}$ speedup using 32 threads compared to the performance of the sequential run. We evaluated the performance impact of the second 
Table 2: Mycoplasma genitalium reaction examples covered by each approach.

\begin{tabular}{|c|}
\hline Reaction examples \\
\hline Approach i - General metabolic reactions: \\
\hline$A T P+A M P \Rightarrow 2 A D P$ \\
\hline Approach ii - Polymer degradation: \\
\hline 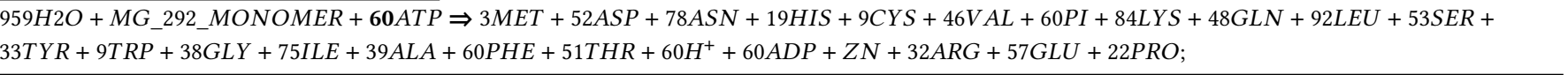 \\
\hline Approach iii - Polymer chain elongation: \\
\hline 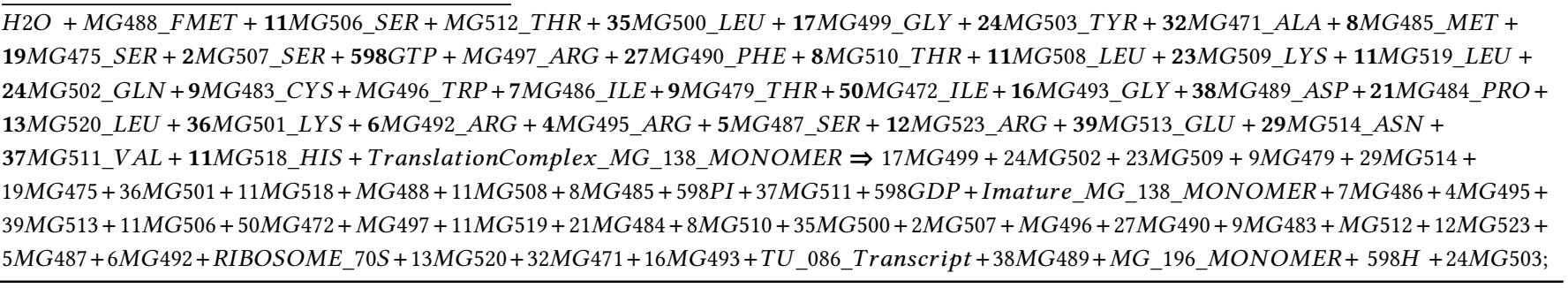 \\
\hline
\end{tabular}

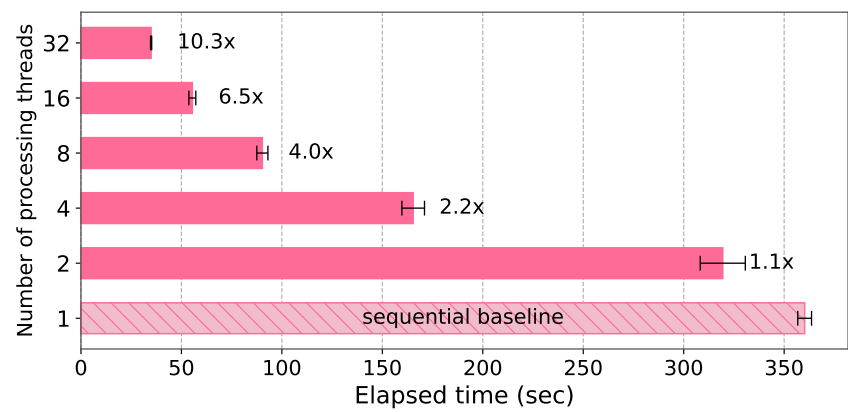

Figure 3: Performance comparison between the parallel and the sequential implementations of NRM. The former relies on the OpenMP parallel construct as shown in Algorithm 2.

level parallelizations described in Section 4.4 by running each of them with and without the first level parallelization using the MG network. We observed that the impact was negligeable. Thus, we used the first-level parallelization only in this study with the MG network. We plan to perform further investigation using different networks with varying connectivity in the future.

5.1.2 Performance comparison against the state-of-the-art. COPASI is a widely used state-of-the-art simulation tool that offers not only the stochastic simulation but also the ODE and the hybrid methods. We used the NRM to compare the performance of COPASI and that of our sequential implementaion. We enabled JIT for COPASI. Note that COPASI does not yet support some of arithmetic operators, such as min, defined in SBML level 3 version 2 specification. Therefore, for the performance evaluations we avoided using the min operator as appeared in Equation 9 and 10 by replacing it with the value calculated at initialization. We created four subnetworks of the MG network by grouping reactions of each approach that is used to construct rate formulas described in Section 4.5 into a subnetwork (subnet i, ii, and ii), and by combining those of approach ii and iii into one (subnet ii+iii). Each of these has different size
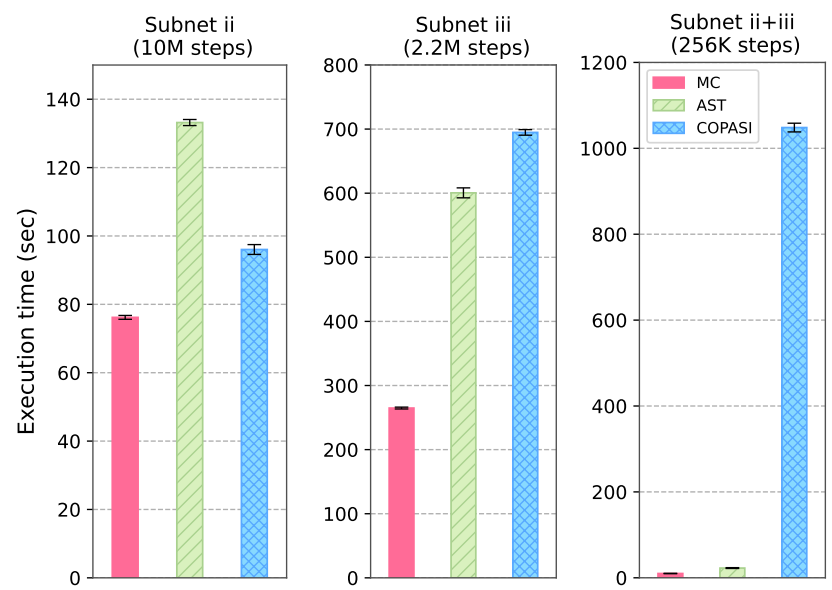

Figure 4: Performance comparison with COPASI. We compare the performance of NRM simulation loop in our sequential implementation with and without JIT enabled, labeled as machine code (MC) and AST respectively, and that in COPASI with JIT enabled.

and characteristics. Figure 4 shows that our implementation outperforms COPASI by a significant margin with JIT enabled for all the subnetworks. COPASI failed to import subnet i due to its large size (see Table 1). Therefore, the case with subnet $\mathrm{i}$ is not included here. COPASI performance degrades significantly with a large model as shown with subnet ii+iii, which only accounts for $19 \%$ of the reactions in the whole network. We ran 10M, 2.2M and 256K simulation steps for subnet ii, subnet iii, and subnet ii+iii respectively. These subnetworks contain reactions that are inter-dependent. Some reactions in a subnetwork may produce the reactants of reactions in another subnetwork. Therefore, we initialized the population states to avoid exhausting reactants by running reactions in isolation, which changed the dynamics as a result. Both COPASI and our implementations used the identical set of models for comparison. 


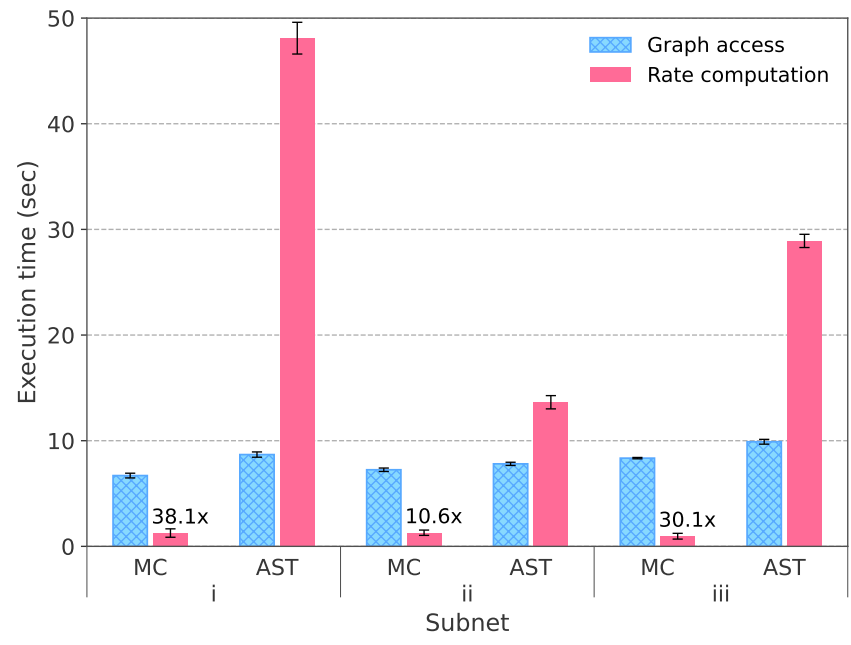

Figure 5: Cost of reaction propensity update. This accounts for the cost of executing line 17 in algorithm 1.

5.1.3 Performance of reaction rate evaluation. In this experiment, we compared the performance of two methods to evaluate the rates of reactions in the MG network. One relies on executing the machine code (MC) generated by the custom JIT compilation and the other on traversing the AST managed by the ExprTk library. We measured the wallclock time to compute the rates of 40 million reactions from each subnet using both methods without updating the population state. This only accounts for a portion of the time to execute a reaction. It does not include the time to update the counts of involved species and the time to update the rate of coupled reactions.

Figure 5 shows the breakdown of the time spent on computing kinetic reaction rates, which includes the times to access the graph for retrieving input states and to calculate the rates. The JIT-based method achieved superior performance compared to the AST-based method with all three subnetworks by the speedup ranging between $10.6 \times$ and $38.1 \times$. ExprTk evaluates a reaction rate by traversing the AST tree it maintains for the expression. On the other hand, the generated machine code does not traverse a tree.

\section{RELATED WORK}

In this section, we provide an overview of the three most commonly used frameworks supporting SBML-based input and implementing stochastic simulation of biochemical systems: COPASI [22], LibRoadRunner [12], and StochKit2 [37]. In addition, we review the existing parallel implementations of SSA.

COPASI supports models written in SBML format, and userfriendly graphical interface [3]. COPASI relies on a third party Just-In-Time (JIT) library, NativeJIT [34], for computing reaction rates using user-provided expressions.

libRoadRunner also supports SBML-based model inputs. It utilizes a custom JIT compiler based on the LLVM [28] framework [12] libRoadRunner allows arbitrary reaction rate expressions.

StochKit2 provides an automatic selection of the optimal SSA implementation between DM [20], ODM [9], and constant-time
SSA [39] based on the input model [37]. The input format is an xmlbased custom format, and provides an SBML-to-StochKit model converter. It supports arbitrary reaction rate functions. StochKit2 offers an option for simultaneous execution of multiple simulations using the Message Passing Interface (MPI) [18].

There exist multiple parallel implementations of SSA variants for shared memory platforms or GPU-based platforms. However, most of these only consider mass-action type of reactions up to 3 rd order (i.e., involving at most two reactants) [23, 26, 27, 29]. As we discuss in Section 3.2, this is often not sufficient for realworld models. Manolakos et al. [29] offers SSA implementation for multi-cores, many-core processors, and FPGA accelerators. It allows the SBML-based input. While it enables the parallelization of a single simulation instance for the first reaction method (FRM), it only supports the coarse-grain parallelization for NRM, distributing realizations of simulation. Komarov et al. [27] implements a variant of the ODM that is amenable to fine-grain parallelization on GPU. LOCAT [23] enables fine-grain parallelism for stochastic simulations using GPUs. Especially, it optimizes the reaction processing by grouping reactions with common reactants and updating the propensities of many reactions in a single operation. STOCHSIMGPU [26] computes ensembles of independent realizations of stochastic simulations on GPU-based platforms. While many of these offer ad hoc methods for simultaneously running independent simulations, modern workflow management system such as Swift/T or Merlin support complex workflows of dependent ensemble jobs requiring less human efforts [14].

\section{CONCLUSION}

The comprehensive computational model of a whole cell enables design and simulation of new in silico experiments and could result in new biological discoveries and insights that could have profound positive implications for fields of biology, medicine, ecology, and bioengineering. Such a model relies on various simulation techniques to computationally represent different components or processes of a biological system. Generation of such advance models present new computational challenges in orchestrating heterogeneous simulations and accelerating them for the problems of unprecedented scales. The stochastic simulation has been an important instrument for studying the time evolution of biochemical systems, especially in cases when counts of critical species in a network is low. However, the computational costs of stochastic simulations are prohibitively expensive for the scale of whole cell model, even for the smallest organism. Moreover, the currently existing tools are not sufficient in that they either do not support general forms of reaction rate formula to accommodate diverse modeling practices in the field, do not parallelize and speed up individual simulation, or do not support the community standard model description. In this work, we have presented a parallel implementation of one of the stochastic simulation algorithms, the next reaction method. Our parallelization approach is uniquely fine-grained while offering the capability to handle general reaction expressions. Such characteristics make it suitable for simulating a large-scale model that integrates multiple sub-models developed with heterogeneous representation approaches and for accelerating simulations of biological timescales. We demonstrated the performance gains in two aspects with a large complex whole 
cell reaction network. One is by partitioning the network and running subnetworks in parallel. The other is by using just-in-time machine code generation. Especially, compared to the state-of-theart, we showed up to 2.6x speedup using a subnetwork that covers $12 \%$ of the whole Mycoplasma genitalium network as well as the superior capacity in handling a large model. We further showed an extra 10x speedup by parallelization on top of the improvement. Tools like this are essential to speed up the simulation of large-scale models as well as to accelerate the development of such models. To the best of our knowledge, this is the first parallel stochastic simulation of the whole cell reaction network of Mycoplasma genitalium. To accommodate future models that are even larger, we plan to expand our framework to distributed memory platforms using optimistic discrete event simulation techniques and to support hybrid simulation methods with ODE. In this work, we also provide a methodology to synthesize representative computational workload for whole cell models. This is not only useful for the development of tools but also potentially for that of models especially when not all parameters are known.

\section{ACKNOWLEDGMENTS}

This work was performed under the auspices of the U.S. Department of Energy by Lawrence Livermore National Laboratory under Contract DE-AC52-07NA27344. LLNL-CONF-819607. We would like to thank Dr. Jonathan Karr and Dr. Arthur Goldberg at the Mount Sinai School of Medicine for their valuable advice.

\section{REFERENCES}

[1] D. Anderson. 2007. A modified next reaction method for simulating chemical systems with time dependent propensities and delays. F. Chem. Phys. 127, 21 (Dec 2007)

[2] L. Bartee, W. Shriner, and C. Creech. [n.d.]. Principles of Biology: DNA Replication in Prokaryotes. https://openoregon.pressbooks.pub/mhccmajorsbio/chapter/dnareplication-in-prokaryotes/.

[3] F. Bergmann, S. Hoops, B. Klahn, U. Kummer, P. Mendes, J. Pahle, and S. Sahle 2017. COPASI and its applications in biotechnology. Journal of biotechnology 261 (2017), 215-220.

[4] M. Blazek, T. Santisteban, R. Zengerle, and M. Meier. 2015. Analysis of fast protein phosphorylation kinetics in single cells on a microfluidic chip. Lab on a Chip 15 3 (2015), 726-734.

[5] OpenMP Architecture Review Boards. [n.d.]. OpenMP. http://www.openmp.org.

[6] B. Bornstein, S. Keating, A. Jouraku, and M. Hucka. 2008. LibSBML: an API library for SBML. Bioinformatics 24, 6 (2008), 880-881.

[7] P. Burke, B. Claudia, L. Costa, and M. Quiles. 2020. A biochemical network modeling of a whole-cell. Scientific reports 10, 1 (2020), 1-14.

[8] Y. Cao, D. Gillespie, and L. Petzold. 2006. Efficient step size selection for the tau-leaping simulation method. 7. Chem. Phys. 124, 4 (Jan 2006).

[9] Y. Cao, H. Li, and L. Petzold. 2004. Efficient formulation of the stochastic simulation algorithm for chemically reacting systems. F. Chem. Phys. 121, 9 (Sep 2004).

[10] A. Riba et al. 2019. Protein synthesis rates and ribosome occupancies reveal determinants of translation elongation rates. Proceedings of the National Academy of Sciences 116, 30 (2019), 15023-15032.

[11] C. Erdem et al. 2020. A Simple and Efficient Pipeline for Construction, Merging, Expansion, and Simulation of Large-Scale, Single-Cell Mechanistic Models. bioRxiv (2020).

[12] E. Somogyi et al. 2015. libRoadRunner: a high performance SBML simulation and analysis library. Bioinformatics 31, 20 (2015), 3315-3321.

[13] J. Karr et al. 2012. A whole-cell computational model predicts phenotype from genotype. Cell 150, 2 (2012), 389-401.

[14] J. Luc Peterson et al. 2019. Merlin: Enabling Machine Learning-Ready HPC Ensembles. Technical Report. LLNL.

[15] L. Xu et al. 2006. Average gene length is highly conserved in prokaryotes and eukaryotes and diverges only between the two kingdoms. Molecular biology and evolution 23, 6 (2006), 1107-1108.

[16] M. Hucka et al. 2003. The systems biology markup language (SBML): a medium for representation and exchange of biochemical network models. Bioinformatics
19,4 (2003), 524-531.

[17] R. Malik-Sheriff et al. 2020. BioModels-15 years of sharing computational models in life science. Nucleic acids research 48, D1 (2020), D407-D415.

[18] MPI forum. [n.d.]. The Message Passing Interface (MPI). https://www.mpiforum.org/.

[19] M. Gibson and J. Bruck. 2000. Efficient exact stochastic simulation of chemical systems with many species and many channels. Journal of Physical Chemistry A 104 (2000), 1876-1889.

[20] D. Gillespie. 1977. Exact stochastic simulation of coupled chemical reactions. F. of Phys. Chem. 81, 25 (Dec 1977), 2340-2361.

[21] Google. [n.d.]. Protocol Buffers. http://developers.google.com/protocol-buffers.

[22] S. Hoops, S. Sahle, R. Gauges, C. Lee, J. Pahle, N. Simus, M. Singhal, L. Xu, P. Mendes, and U. Kummer. 2006. COPASI-a complex pathway simulator. Bioinformatics 22, 24 (2006), 3067-3074.

[23] Sagar Indurkhya and Jacob Beal. 2010. Reaction Factoring and Bipartite Update Graphs Accelerate the Gillespie Algorithm for Large-Scale Biochemical Systems. PLoS ONE 5, 1 (Jan 2010).

[24] J. Karr and A. Goldberg. 2020. An introduction to whole-cell modeling. https://buildmedia.readthedocs.org/media/pdf/intro-to-wc-modeling/latest/ intro-to-wc-modeling.pdf. Accessed: 2021-01-20.

[25] George Karypis and Vipin Kumar. 1998. Multilevel k-way Partitioning Scheme for Irregular Graphs. 7. Parallel Distrib. Comput. 48, 1 (1998), 96-129.

[26] G. Klingbeil, R. Erban, M. Giles, and P. Maini. 2011. STOCHSIMGPU: parallel stochastic simulation for the Systems Biology Toolbox 2 for MATLAB. Bioinformatics 27, 8 (April 2011), 1170-1171.

[27] Ivan Komarov and Roshan M. D'Souza. 2012. Accelerating the Gillespie Exact Stochastic Simulation Algorithm Using Hybrid Parallel Execution on Graphics Processing Units. PLoS ONE 7, 11 (Nov 2012).

[28] C. Lattner and V. Adve. 2004. LLVM: A compilation framework for lifelong program analysis \& transformation. In Intl Symp. on Code Generation and Optimization, 2004. CGO 2004. IEEE, 75-86.

[29] E. Manolakos and E. Kouskoumvekakis. 2017. StochSoCs: High Performance Biocomputing Simulations for Large Scale Systems Biology. (2017), 921-928.

[30] J. McCollum, G. Peterson, C. Cox, M. Simpson, and N. Samatova. 2006. The sorting direct method for stochastic simulation of biochemical systems with varying reaction execution behavior. Comput. Biol. Chem. 30, 1 (Feb 2006), 39-49.

[31] Ron Milo and Rob Phillips. [n.d.]. Cell biology by the numbers: How big is the average protein? http://book.bionumbers.org/how-big-is-the-average-protein/.

[32] Ron Milo and Rob Phillips. [n.d.]. Cell biology by the numbers: How fast do RNAs AND proteins degrade? http://book.bionumbers.org/how-fast-do-rnasand-proteins-degrade/.

[33] Ron Milo and Rob Phillips. [n.d.]. Cell biology by the numbers: what is faster transcription or translation? http://book.bionumbers.org/what-is-fastertranscription-or-translation/.

[34] NativeJIT. [n.d.]. https://github.com/BitFunnel/NativeJIT. Accessed: 2021-02-13.

[35] A. Partow. [n.d.]. C++ Mathematical Expression Toolkit Library (ExprTk). https: //github.com/ArashPartow/exprtk.

[36] Conner I. Sandefur, Maya Mincheva, and Santiago Schnell. 2013. Network representations and methods for the analysis of chemical and biochemical pathways. Mol Biosyst. 9, 9 (Sept 2013), 2189-2200.

[37] K. Sanft, S. Wu, M. Roh, J. Fu, R. Lim, and L. Petzold. 2011. StochKit2: software for discrete stochastic simulation of biochemical systems with events. Bioinformatics 27, 17 (2011), 2457-2458.

[38] C Sayikli and E. Bagci. 2011. Limitations of using mass action kinetics method in modeling biochemical systems: illustration for a second order reaction. In Intl. Conf. on Computational Science and Its Applications. Springer, 521-526.

[39] Alexander Slepoy, Aidan P Thompson, and Steven J Plimpton. 2008. A constanttime kinetic Monte Carlo algorithm for simulation of large biochemical reaction networks. The journal of chemical physics 128, 20 (2008), 05B618.

[40] A. van der Schaft, S. Rao, and B. Jayawardhana. 2013. On the mathematical structure of balanced chemical reaction networks governed by mass action kinetics. SIAM 7. Appl. Math. 73, 2 (2013), 953-973.

[41] J. Yeom, K. Georgouli, R. Blake, and A. Navid. [n.d.]. Whole Cell Simulator. https://github.com/LLNL/wcs. 